\title{
Application of Intelligent Equipment on Novel Coronavirus Pneumonia Designated Hospital
}

\author{
Lili Li'1, Weiqiang Gan'1, Zimei Zheng1, Shuxian Jiang1, Lihua Zheng1, Miaoxia Chen², \\ Juan Wang1, Na He1, Zhiliang Gao ${ }^{1 *}$, Yuanli Chen ${ }^{*}$ \\ ${ }^{1}$ Department of Infectious Diseases, The Third Affiliated Hospital of Sun Yat-sen University, Guangzhou, China \\ ${ }^{2}$ Nursing Department, the Third Affiliated Hospital of Sun Yat-sen University, Guangzhou, China \\ Email:22017377@qq.com, ganweiqiang@aliyun.com,1065388297@qq.com,1103071055@qq.com,306167269@qq.com, \\ 1109986151@qq.com,1061613246@qq.com,282222923@qq.com, ${ }^{*}$ gaozhl@mail.sysu.edu.cn, ${ }^{*}$ cyl.ff@163.com
}

How to cite this paper: Li, L.L., Gan, W.Q., Zheng, Z.M., Jiang, S.X., Zheng, L.H., Chen, M.X., Wang, J., He, N., Gao, Z.L. and Chen, Y.L. (2020) Application of Intelligent Equipment on Novel Coronavirus Pneumonia Designated Hospital. Open Journal of Nursing, 10, 770-776. https://doi.org/10.4236/ojn.2020.108055

Received: July 1, 2020

Accepted: August 25, 2020

Published: August 28, 2020

Copyright $\odot 2020$ by author(s) and Scientific Research Publishing Inc. This work is licensed under the Creative Commons Attribution International License (CC BY 4.0)

http://creativecommons.org/licenses/by/4.0/

\begin{abstract}
Objective: To explore the application of intelligent equipment in non-negative pressure isolation ward for COVID-2019 patients. Method: From February 1 to March 17, 2020, intelligent equipment, such as communication interaction system, intelligent disinfection robot, delivering robot, were used in nonnegative pressure isolation ward of COVID-2019. With the help of communication interaction system to supervise the implementation of infection prevention and control, and observe the incorrect situation of pee use and personal behavior before and after the implementation. The disinfection robot and meal delivery robot were used in ward disinfection and life nursing combined with nursing practice. Result: Through the supervision of communication interaction system, the frequency of pee use and personal behavior was reduced. The frequency of bad articles before and after improvement was wearing protective clothing $(2.80 \% / 0.84 \%)$, taking off protective clothing (5.87\%/0.84\%), personal behavior observation (8.38\%/1.90\%), $\mathrm{P}<0.01$. The robot disinfected and delivered medicine for $912.5 \mathrm{~h}$, saving 225 shifts of nursing staff. Conclusion: Intelligent equipment is a good option for infection control in isolation ward of COVID-2019. It can not only reduce the workload of health workers, but also the cross-infection.
\end{abstract}

\section{Keywords}

Intelligent Equipment, COVID-2019, Non-Negative Pressure Isolation Ward

\section{Introduction}

COVID-19 (Corona Virus Disease 2019) has been widely affecting many regions globally, the number of confirmed cases is still increasing. The medical and 
health institutions are facing great challenges because of the severe epidemic situation and strong infectivity of the novel coronavirus (SARS-CoV-2), which call for high requirements of medical treatment technology, medical environment, manpower and material allocation. As the first-line battlefield for treatment, the isolation ward particularly has become the vital and difficult management area for the prevention and control of COVID-2019. As a provincial infectious disease hospital, we were designated for admitting COVID-2019 confirmed or suspected cases. Due to time constraints and limited conditions, isolation ward was built on Jan 19, 2020, and patients were admitted on January 22.

Intelligent equipment has been developing in clinical nursing, ward and material management. It can not only meet the needs of difficult nursing, but also improve the efficiency of nursing [1] [2] [3]. Therefore, we explored the application of intelligent equipment in isolation ward. Since February 1 to March 17, 2020, communication interaction system, disinfection robot and delivering robot were introduced to assist infection control and nursing work.

\section{Method}

\subsection{Supervise the Prevention and Control of Hospital Infection through Communication Interactive System}

The task of fighting the epidemic was rush and heavy. Due to the emergent situation of soaring patients, medical staff were very vulnerable to infection. How to avoid infection caused by inadequate prevention and control measures was a big challenge. Due to less use and specialized training at ordinary times, there is a rush and tight time after the outbreak of the epidemic, so the training can not accurately master and improve personal protection skills in a short period of time. During the Ebola outbreak, it was found that the video monitoring system can be used for infection control supervision and real-time reminder to prevent cross-infection [4].

Therefore, our hospital used the communication interaction system, namely video monitoring intercom system, to supervise the infection prevention and control, and observed the incorrect use of pee and personal behavior of medical staff in isolation ward through the self-made infection prevention and control supervision form. The sense and control supervisor shall supervise and record through two forms of normal random and regular inspection. Compare the situation before and after using the communication interaction system.

\subsubsection{Formulate the Infection Prevention and Control Supervision Form}

We refer to novel coronavirus infection prevention and control technical guidelines for medical institutions first edition and isolation specification of hospitals WS/T311-2009. In view of the problems existing in the implementation of the nursing work flow in the isolation ward. The infection prevention and control supervision form was formulated by the hospital infection department and nursing department, combined with the actual situation of hospital nursing work process and disinfection and isolation quality standards. The form con- 
tained three parts, the first part was wearing and removing personal protective equipment, composed of 11 and 22 steps respectively. The second part was personal behavior observation, including 21 items, mainly to observe whether high-risk actions at bedsides were safe, whether there was occupational exposure risk during medical operation. Cleaning and disinfecting methods, medical waste disposal ways were also inspected. The third part was the process observation. By using the communication interaction system, the infection control monitor supervised the process randomly or regularly through the following three ways.

\subsubsection{Supervision of Infection Prevention and Control through Communication Interaction System}

1) Supervise and standardize the use of PPE. The use of PPE was vital to prevent cross-infection especially during the removal [4]. By using this system, the monitor supervised the wearing and removal of PPE through the checklist. When the staff entered the isolation ward for the first time, the monitor would remind the staff of each step, recorded the irregularities on the checklist, gave them timely voice remind, and then gave correct guidance when they came out to the clean area. After that, the monitor would conduct another $2-3$ rounds of random inspection on them.

2) Monitor the implementation of prevention and control in the contaminated area. The monitor watched the work of the medical staff in the isolation ward through real time surveillance, and paid special attention to non-medical staff such as cleaning workers. When the staff entered the isolation ward for the first time, the monitor would check their working conditions throughout the whole process, recorded in the checklist, corrected the inappropriate behavior timely, and gave them further guidance.

3) Supervise the development of novel workflow. Due to the continuous adjustment of COVID-2019 management, there were new work assignments. In order to ensure the smooth completion of the work, we used the system to check the implementation of the new work, recorded in the checklist where the procedure was not smooth, and improved the work process accordingly.

\subsection{Disinfect the Ward by Disinfection Robot}

COVID-2019 is highly infectious. It can be inactivated by ultraviolet, $75 \%$ alcohol, chlorine containing disinfectant, peroxide acetic acid [5]. Disinfection is an important measure to prevent cross-infection, especially in isolation ward. According to China COVID-2019 prevention and control plan [5], in non-negative pressure isolation ward, air sterilizing machine is required when people are in the room, otherwise ultraviolet disinfection can be used. Terminal disinfection is carried out by spray disinfection using $0.5 \%$ peracetic acid or $3 \%$ hydrogen peroxide disinfectant with no dead angle or space. However, the isolation ward was temporarily reconstructed due to the short time and limited conditions. What's more, it was non-negative pressure type, and the disinfection device was limited, which made disinfection difficult. In order to ensure the disinfection effect of 
isolation ward, we explored the application of disinfection robot to assist disinfection work.

1) Air disinfection. We conducted routine air disinfection in the contaminated and buffer area, the room was disinfected by air disinfector. In public area, the ultraviolet lamp was used for automatic disinfection at fixed time and location. The disinfection time of each location was 1 hour per time, 4 times per day. The disinfection route was: doctor office $\rightarrow$ nurse station $\rightarrow$ corridor $\rightarrow$ hygiene room.

2) Terminal disinfection. For terminal disinfection, the robot carried out the disinfection using ultraviolet light plus ultra-dry mist hydrogen peroxide combination mode, the nurse remotely controlled the robot, which automatically calculated the ward space, and gave aerosol spray disinfection with the amount of 20 - 30 milliliters per cubic meters. The disinfection order was from up to down, left to right, spray was evenly on the surface and space, disinfect time was 60 minutes. After completion, the cleaner would use $1000 \mathrm{mg} / \mathrm{L}$ chlorine containing disinfectant to wipe the ground, and mopped it with clean water after 30 minutes.

\subsection{Assistance of Daily Life Care by Delivering Robot}

In the isolation ward, the nursing staff should send three meals a day and daily necessities to the patients. Since patients were suddenly isolated for treatment because of the disease, or persons with close contacts may also be isolated for observation, they were unable to prepare daily necessities, so nurses were often required to deliver daily necessities. This part of the workload was quite large and occupied a lot of nursing time. In order to enable nurses to have more energy to carry out treatment or nursing operations, and better meet the needs of patients, we utilized delivering robot to finish this job. Nurses set robots to deliver meals or items as required. The delivery of objects included three methods. The first was "point-to-point" delivery. For meal delivery, the nurse placed the food on the robot shelf in the buffer room, set the order and the robot delivered the food accordingly. The robot would remind the patient to fetch the food. Once food delivery was completed, the robot returned to the buffer room for next task. The second was "call delivery". It was used to handle the delivery of daily necessities for patients. The last was "patrol mode". The nurse set up a robot twice a day to remind the patient to measure temperature, drink more water and so on.

\section{Result}

\section{No nosocomial infection occurred}

From February 1 to March 16, 2020, the medical staff and staff in the isolation ward were not infected.

Reduce the mistakes of using PPE and personal behavior through communication interaction system 
Using self-made checklist, the sensory and control supervisor inspects the incorrect use of pee and personal behavior before and after using the communication interaction system. From February 1 to March 17, 2020, a total of 65 isolation ward staff were observed, including 15 doctors, 47 nurses and 3 workers. Use communication interaction system to inspect the changes before and after improvement (Table 1). Before utilizing intelligent equipment, the incidence of misconduct during wearing, removing personal protective equipment, other inappropriate misconducts were $2.80 \%, 5.87 \%, 8.38 \%$ respectively while it reduced to $0.84 \%, 0.84 \%, 1.90 \%$ after using intelligent equipment. At the same time, through the communication interaction system, we found that there were 23 items in the process that were not smooth, including the specimen storage process, the patient discharge process and so on, we improved them one by one.

\section{The robots efficiently finished the disinfection and delivery task}

There was less than one nurse per shift after using the robot than before. From February 1 to March 17, 2020, the disinfection robot completed 180 air disinfection tasks, 84 terminal disinfection tasks, with a total disinfection time of 804 hours. The delivering robot completed 155 meal delivering tasks, 98 daily necessities delivering tasks, with a total working time of 108.5 hours. The total working time of disinfection and delivery is $912.5 \mathrm{~h}$, which is equivalent to the working time of 225 nursing staff if it is calculated according to the working hours of nurses in each shift.

\section{Discussion}

COVID-19 is a new highly infectious disease. The prevention and control of infection in isolation ward is the key point in fighting the epidemic. Through video monitoring system, we developed detailed quality control form by evaluating the key points of infection occurrence, which included wearing and removing PPE for medical staff during the operation and process, the common mistakes were immediately corrected and improved, which ensure high quality operation and smooth workflows among medical staff. More importantly, through the intercom system, improper operation was timely corrected, which could effectively prevent the occurrence of infection. In addition, the intelligent robot can finish some disinfection and daily life care work, reduce the working time and unnecessary contact of nurses with patients in the contaminated area, thus cut down the chance of cross-infection to a certain extent. It can be seen that the use of intelligent equipment is conducive to the prevention and control of infection in

Table 1. The frequency of incorrectness before and after supervision.

\begin{tabular}{ccccc}
\hline Items & Before supervision & After supervision & $\mathrm{X}^{2}$ & $\mathrm{p}$ \\
\hline Wearing PPE & $2.80 \%(20 / 715)$ & $0.84 \%(6 / 715)$ & 14.873 & $<0.001$ \\
Removing PPE & $5.87 \%(84 / 1430)$ & $0.84 \%(24 / 2860)$ & 56.974 & $<0.001$ \\
Personal Behavior & $8.38 \%(44 / 525)$ & $1.90 \%(10 / 525)$ & 22.568 & $<0.001$ \\
\hline
\end{tabular}


isolation ward. In future, we can apply more advanced artificial intelligence technology, such as Internet of Things technology [6], identified with RFID as the method of communication, to achieve all-round, all-time monitoring work, so as to discover, intelligently pre-warn and effectively prevent and control infection more quickly.

Disinfection work in isolation wards for COVID-2019 patient is complicated, which needs not only regular routine ultraviolet disinfection, but also hydrogen peroxide spray for terminal disinfection. The workload is heavy and time-consuming, and disinfection environment is not suitable for people hanging around, which is a big challenge for nursing staff. On the contrary, the disinfection robot can complete these disinfection works more efficiently. At the same time, the robot can also assist the nursing staff to deliver food and materials, reduce the working hours for non-core treating or nursing, save more energy for nurses to carry out important core treating or nursing procedures, reduce manpower consumption, and make more efficient use of human resources. At present, the application of robots in the nursing industry is gradually increasing, such as objects delivery, patient transport, diet care, elderly care [7] [8] and so on. It can save nursing resources and provide higher quality services [8]. However, at present, robots are seldom used in infectious disease care in China. Robot nursing has more advantages in dealing with severe infectious diseases. In future, robot nursing needs more exploration and application. As a user, nurses should actively participate in the research design to make intelligent devices more suitable for clinical use.

\section{Intelligent equipment can protect the safety of isolation ward staff}

Medical staff may feel sick in the contaminated area, the video monitoring system can dynamically help to inspect the working status of the medical personnel, any unfitness of the medical personnel would be noticed for next step management so as to avoid accident. Medical staff can communicate with patients more efficiently through video intercom system, such as answering patients' questions, guiding patients to measure body temperature, so as to reduce unnecessary contact between medical staff and patients and effectively reduce the chance of medical infection.

In conclusion, the application of intelligent equipment in non-negative pressure isolation ward is easier to meet the needs of patients, make the implementation of infection control more efficient, save manpower and protection resources. Therefore, it can avoid cross-infection in the hospital, improve safety and work efficiency. Due to the urgency of ward establishment and lack of experience, the control measures and effect observation have some limitations. For example, the monitoring data of the isolation ward before and after the use of intelligent equipment, such as surface disinfection and air disinfection, were not monitored. Moreover, the application of intelligent equipment is limited and the use time is short. There are still many unpredictable situations, which can only provide a reference for the intelligent management of isolation ward in the fu- 
ture. However, with more participation in various disciplines, the management of the isolation ward by intelligent equipment will be more scientific and perfect. It is hoped that a set of perfect management modes of the isolation ward will be formed in future works to ensure the physical and mental safety of medical staff, reduce the consumption of medical materials, and provide more humanized care for patients.

\section{Conflict of Interest}

No any editorial or financial conflict of interest exists.

\section{References}

[1] Zhou, Y. and Li, Z. (2018) Progress of Application of Artificial Intelligence Technology in Elderly Care. Chinese Nursing Management, 18, 777-780.

[2] Du, Y.Y., Dong, D.H., Ma, F., et al. (2019) Progress of Application of Artificial Intelligence in Nursing. Nursing Journal of Chinese People's Liberation Army, 36, 58-61.

[3] Tu, A.Q. and Zeng, T.Y. (2020) Application and Prospect of Artificial Intelligence Technology in Clinical Nursing. Nursing Research, 34, 269-272.

[4] Li, P., Li, J.Q., Zhan, Y., et al. (2016) Comparison of Wearing and Removing Ways of Personal Protective Equipment between Liberia-Cina Ebora. Chinese Nursing Research, 30, 1397-1399.

[5] National Health Commission of the People's Republic of China (2020) Notice on the Novel Coronavirus Infection Prevention and Control Plan (Seventh Trial Version). National Health Office Disease Control Letter [2020]108.

[6] Yao, H.W., Liu, B.W., Suo, J.J., et al. (2017) Progress of Application of Internet of Things Technology in Management of Nosocomial Infections. Chinese Journal of Nosocomiology, 27, 3131-3134.

[7] Huang, D.Z., You, Q.X., Hang, Z.G., et al. (2013) Design of Nurse Assistant Robot. Machine Tools and Hydraulics, 41, 31-41.

[8] He, Y. and Li, L. (2018) Research Progress on Application of Robots in Nursing. Chinese Journal of Nursing, 53, 1140-1143. 